\title{
Modelling of hydro-thermo-chemo-mechanical phenomena in building materials
}

\author{
D. GAWIN ${ }^{1 *}$ M. KONIORCZYK ${ }^{1}$, and F. PESAVENTO ${ }^{2}$ \\ ${ }^{1}$ Department of Building Physics and Building Materials, Lodz University of Technology, 6 Politechniki Ave., 90-924 Lodz, Poland \\ ${ }^{2}$ Department of Civil, Architectural and Environmental Engineering, University of Padova, Via Marzolo 9, 35131 Padova, Italy
}

\begin{abstract}
A general approach to modelling chemical degradation processes in building materials, due to combined action of variable hydrothermal, chemical and mechanical loads, is presented. Mechanics of multiphase porous media and damage mechanics are applied for this purpose, and kinetics of degradation processes is described with evolution equations based on thermodynamics of chemical reactions. The mass-, energy - and momentum balances, as well as the evolution equations, constitutive and physical relations are briefly summarized. Then, the model governing equations are numerically solved with the finite element method. Three examples of the model application for analyzing degradation processes of building materials are presented and discussed. The first one deals with capillary suction of the salt solution by two different building materials, the second one with the salt crystallization during drying of a brick wall, and the third one concerns calcium leaching from a concrete wall due to the chemical attack of pure water under pressure gradient at two different temperatures.
\end{abstract}

Key words: building materials, chemical degradation, hydro-thermal processes, mechanics of multi-phase porous media, numerical solution.

\section{List of main symbols}

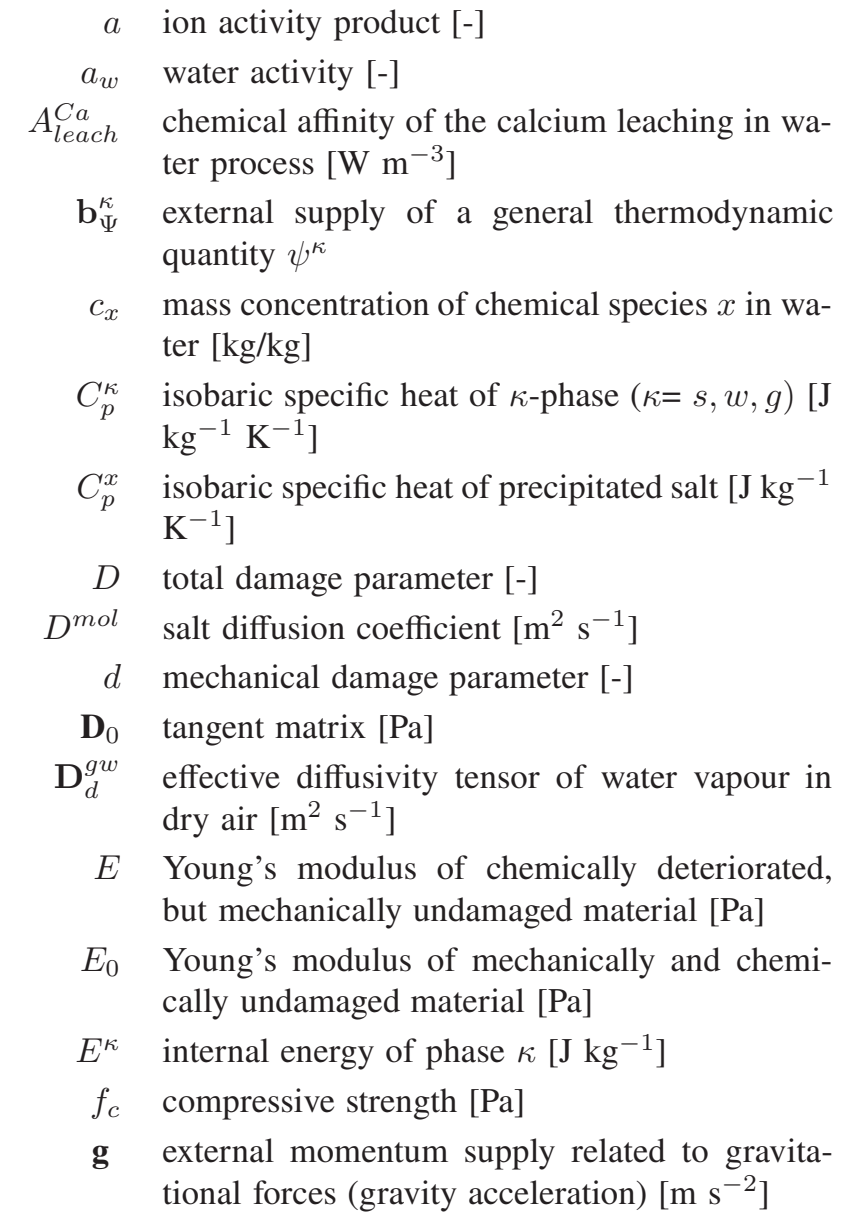

$\mathbf{G}_{\Psi}^{\kappa} \quad$ net production of thermodynamic quantity $\psi^{\kappa}$

$H^{g w}$ enthalpy of water vapour $\left[\mathrm{J} \mathrm{kg}^{-1}\right]$

$H^{w}$ enthalpy of water [ $\mathrm{J} \mathrm{kg}^{-1}$ ]

$H_{w}^{x} \quad$ enthalpy of $x$ chemical species dissolved in water $\left[\mathrm{J} \mathrm{kg}^{-1}\right]$

$H_{p}^{x} \quad$ enthalpy of precipitated $x$ chemical species $\left[\mathrm{J} \mathrm{kg}^{-1}\right]$

$H_{s}^{x} \quad$ enthalpy of $x$ chemical species in skeleton [ $\mathrm{J} \mathrm{kg}^{-1}$ ]

$h^{\kappa} \quad$ specific heat source in the bulk phase $\kappa$ [W kg-1]

$\mathbf{i}_{\Psi}^{\kappa} \quad$ flux vector associated with thermodynamic quantity $\psi^{\kappa}$

I second order unit tensor [-]

$J_{w g}^{w} \quad$ curvature of water / gas interface $\left[\mathrm{m}^{-1}\right]$

$\mathbf{J}_{d}^{g w}$ diffusive flux of vapour $\left[\mathrm{kg} \mathrm{m}^{-2} \mathrm{~s}^{-1}\right]$

$\mathbf{J}_{d}^{g a}$ diffusive flux of dry air $\left[\mathrm{kg} \mathrm{m}^{-2} \mathrm{~s}^{-1}\right]$

$\mathbf{J}_{D}^{x} \quad$ dispersive flux of chemical species $x[\mathrm{~kg}$ $\mathrm{m}^{-2} \mathrm{~s}^{-1}$ ]

k absolute permeability tensor $\left[\mathrm{m}^{2}\right]$

$k$ absolute permeability (scalar) $\left[\mathrm{m}^{2}\right]$

$K_{x} \quad$ equilibrium constant for precipitated / dissolved salt $x[-]$

$k^{r \kappa}$ relative permeability of $\kappa$-phase $(\kappa=$ $g, w)[-]$

$m_{M}, m_{X} \quad$ molality of cations, anions [mol kg-1]

$\dot{m}_{\text {vap }}$ rate of mass due to phase change $\left[\mathrm{kg} \mathrm{m}^{-3}\right.$ $\mathrm{s}^{-1}$ ]

*e-mail: Dariusz.Gawin@p.lodz.pl 


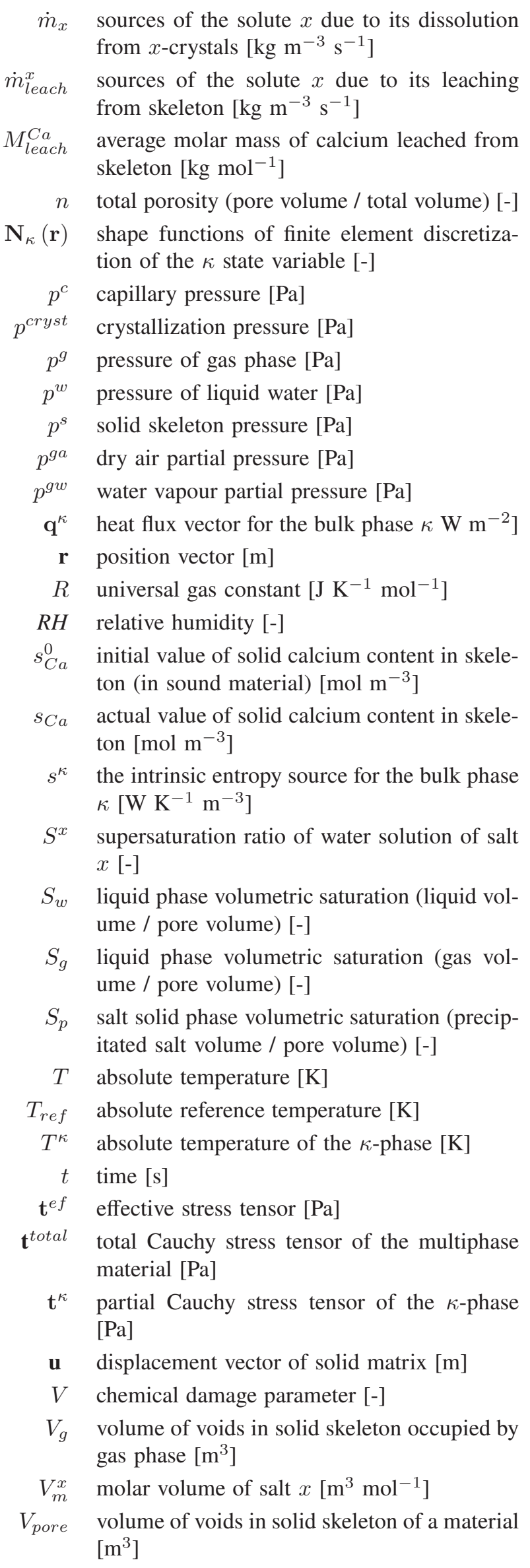

$V_{w} \quad$ volume of voids in solid skeleton occupied by liquid phase $\left[\mathrm{m}^{3}\right]$

$V_{p} \quad$ volume of voids in solid skeleton occupied by precipitated salt $\left[\mathrm{m}^{3}\right]$

$\mathbf{v}^{\kappa} \quad$ local velocity of the $\kappa$-phase $(\kappa=g, w, s)$ [m $\mathrm{s}^{-1}$ ]

$\mathbf{v}^{g, s} \quad$ relative velocity of gaseous phase with respect to the skeleton $\left[\mathrm{m} \mathrm{s}^{-1}\right]$

$\mathbf{v}^{w, s}$ relative velocity of liquid phase with respect to the skeleton $\left[\mathrm{m} \mathrm{s}^{-1}\right]$

$\mathbf{X}$ vector of nodal values of state variables, $\overline{\mathbf{p}}^{g}$, $\overline{\mathbf{p}}^{c}, \overline{\mathbf{T}}, \overline{\mathbf{c}}_{x}, \overline{\mathbf{u}}$ in finite element space discretization

$\alpha_{c}$ convective heat exchange coefficient [W $\mathrm{W}^{-2}$ $\mathrm{K}^{-1}$ ]

$\beta_{c} \quad$ convective mass exchange coefficient $\left[\mathrm{m} \mathrm{s}^{-1}\right.$ ]

$\Delta H_{\text {vap }} \quad$ enthalpy of vaporization $\left[\mathrm{J} \mathrm{kg}^{-1}\right]$

$\Delta H_{\text {prec }}^{x} \quad$ enthalpy of precipitation / dissolution of salt $x$ in water $\left[\mathrm{J} \mathrm{kg}^{-1}\right]$

$\Delta H_{\text {leach }}^{x} \quad$ enthalpy of leaching chemical species $x$ from skeleton $\left[\mathrm{J} \mathrm{kg}^{-1}\right]$

$\Delta t \quad$ time step length in finite difference scheme [s]

$\varepsilon_{t o t}$ total strain tensor [-]

$\mathcal{E}_{c} \quad$ creep strain tensor [-]

$\varepsilon_{c h} \quad$ chemical strain tensor [-]

$\varepsilon_{t} \quad$ thermal strain tensor [-]

$\varphi^{\kappa} \quad$ volumetric entropy increase of the $\kappa$-phase [J $\mathrm{m}^{-3} \mathrm{~K}^{-1}$ ]

$\boldsymbol{\Phi}^{\kappa} \quad$ entropy flux of the $\kappa$-phase [W $\mathrm{K}^{-2} \mathrm{~m}^{-2}$ ]

$\gamma_{M}, \gamma_{X}$ activity coefficient of cations, anions [-]

$\gamma^{w g} \quad$ surface tension of water / gas interface $\left[\mathrm{J} \mathrm{m}^{-2}\right]$

$\Gamma_{\text {leach }}^{C a}$ degree of calcium leaching from material skeleton [-]

$\Gamma_{\text {leach }}^{x}$ degree of $x$-species leaching from material skeleton [-]

$\chi_{s}^{w s} \quad$ skeleton surface fraction in contact with liquid water [-]

$\chi_{s}^{c s}$ skeleton surface fraction in contact with salt crystals [-]

$\kappa$ equilibrium function for calcium leaching [W $\mathrm{mol}^{-1}$ ]

$\chi_{e f}$ effective thermal conductivity of multiphase material $\left[\mathrm{W} \mathrm{K}^{-1} \mathrm{~m}^{-1}\right.$ ]

$\nu \quad$ Poisson's ratio [-]

$v_{M}, v_{X}, v_{0}$ stoichiometric coefficients [-]

$\psi^{\kappa}$ a general thermodynamic property of the $\kappa$ phase

$\sigma_{x}$ reflection coefficient for chemical species $x$ (degree of skeleton semi-permeability) [-] 


\author{
$\Pi^{f} \quad$ disjoining pressure $[\mathrm{Pa}]$ \\ $\rho^{\kappa} \quad$ real density of the $\kappa$-phase $(\kappa=g, w, s, g a$, \\ $g w)\left[\mathrm{kg} \mathrm{m}^{-3}\right]$ \\ $\rho^{x} \quad$ density of precipitated chemical species $x[\mathrm{~kg}$ \\ $\mathrm{m}^{-3}$ ]
$\left(\rho C_{p}\right)_{e f} \quad$ effective, isobaric volumetric heat of porous medium $\left[\mathrm{J} \mathrm{m}^{-3} \mathrm{~K}^{-1}\right.$ ]
$\tau_{\text {leach }}^{\text {Ca }}$ characteristic time of calcium leaching in wa- ter $[\mathrm{s}]$.

\section{Mathematical symbols \\ $(\cdot)^{T}$ tensor (matrix) transposition operator \\ $\frac{D^{s}(\cdot)}{D t}$ material time derivative with respect to skele- ton, e.g. $\frac{D^{s} f^{\kappa}}{D t}=\frac{\partial f^{\kappa}}{\partial t}+\mathbf{v}^{\kappa s} \cdot \operatorname{grad} f^{\kappa}$} \\ $\operatorname{grad}(\cdot) \quad$ gradient operator \\ $\operatorname{div}(\cdot)$ divergence operator.
}

\section{Introduction}

A possible accurate prediction of degradation processes of building structures during their whole life cycle, at normal service conditions and after exposition to a chemically aggressive environment, is of great practical importance. Much research has been devoted to this subject in recent decades, and several predictive mathematical models have been developed, e.g. for analysis of chemical degradation of concrete structures due to alkali silica reaction [1-4]. At the Lodz University of Technology and the University of Padova research on the modelling of building materials as a multiphase, porous medium has been carried out for many years. Concepts of mechanics of porous media and thermodynamics of chemical reactions have been adopted for modelling heat, mass and momentum transport and chemical processes in cement based materials, e.g. [5-15], including also effects of water sorption hysteresis [16]. Previously this approach has been successfully applied to the numerical analysis of such difficult problems as hygro-thermo-chemical behaviour of concrete at early ages or degradation at high temperature, giving results in good agreement with experimental data, [5-8]. Here, a general approach to mathematical modelling degradation processes of building materials (including cement based materials which are particularly difficult for modelling), due to combined action of hydro-thermal, chemical and mechanical loads, is presented. Mechanics of multiphase porous media, see e.g. [17-19], is applied for this purpose. The mass-, energy- and momentum balances, evolution equations, as well as some physical and constitutive relationships are briefly presented, and then numerically solved with the finite element method. Three examples of the model application for analysis of ions transport and chemical degradation processes of building materials in various hydro-thermal conditions are presented and discussed. The first one concerns capillary suction of the salt solution by two different building materials, the second one the salt crystallization during drying of a brick wall, and the third one deals with calcium leaching in a concrete wall exposed to chemical attack of pure water under pressure gradient at two different temperatures.

\section{Mathematical model development}

Development of the mathematical model proposed by the authors for analyzing chemical degradation of building materials is presented in this section. The model is based on the mechanics of multiphase porous media [17-19], and considers chemical reactions and phase changes, as well as most important material nonlinearities and mutual couplings between different physicochemical processes in the material. Main stages of the mathematical model development are briefly presented in successive subsections.

In modelling it is usually assumed that the material components are in thermodynamic equilibrium state locally. In this way their thermodynamic state can be described by one common set of state variables and not by separate ones. In development of the present model we assume that a porous material is in hygro-thermal equilibrium state locally, but there exists chemical non-equilibrium. The material is considered as a multiphase medium where the voids of the solid skeleton (with volume $V_{\text {pore }}$ ) are filled with various combinations of liquid- and gas- and solid (precipitated salt) phases. The fluids filling pore space are the moist air (i.e. mixture of dry air and vapour), capillary water and physically adsorbed water, occupying the volumes: $V_{g}$ and $V_{w}$, respectively. The precipitated salt crystals constitute the solid phase which is present in pore space and occupies the volume $V_{p}$. Defining the pore saturation degree with the $\kappa$-phase, $S_{\kappa}=V_{\kappa} / V_{\text {pore }}(\kappa=w, g, p)$, and considering an obvious relationship, $V_{\text {pore }}=V_{w}+V_{g}+V_{p}$, one obtains the following relation, $S_{w}+S_{g}+S_{p}=1$.

2.1. Microscopic conservation equations. In the present approach the mathematical model is formulated by using two different scales starting from micro-level, i.e. from a local form of the governing equations at the pore scale. The microscopic situation of any $\kappa$-phase of the considered medium is described by the classical equations of continuum mechanics. At the interfaces with other constituents, the material properties and thermodynamic quantities may present step discontinuities.

For a thermodynamic property in a point with a given position $\mathbf{r}$ and at time instant $t, \psi^{\kappa}(\mathbf{r}, t)$, the balance equation within the $\kappa$-phase may be written as follows [18],

$$
\frac{\partial\left(\rho^{\kappa} \psi^{\kappa}\right)}{\partial t}+\operatorname{div}\left(\rho^{\kappa} \psi^{\kappa} \mathbf{v}^{\kappa}\right)=\operatorname{div} \mathbf{i}_{\Psi}^{\kappa}+\rho^{\kappa} \mathbf{b}_{\Psi}^{\kappa}+\rho^{\kappa} \mathbf{G}_{\Psi}^{\kappa},
$$

where $\rho^{\kappa}(\mathbf{r}, t)$ and $\mathbf{v}^{\kappa}(\mathbf{r}, t)$ are the actual values (at time instant $t)$ of density and velocity field of the $\kappa$-phase in a fixed point $\mathbf{r}$ in space, $\mathbf{i}_{\Psi}^{\kappa}(\mathbf{r}, t)$ is the flux vector associated with $\psi^{\kappa}, \mathbf{b}_{\Psi}^{\kappa}(\mathbf{r}, t)$ the external supply of $\psi^{\kappa}$ and $\mathbf{G}_{\Psi}^{\kappa}(\mathbf{r}, t)$ is the net production of $\psi^{\kappa}$. All the quantities in Eq. (1) are related to the phase which is physically present in the point $\mathbf{r}$ of porous medium, Fig. 1. Fluxes are positive as outflows. 


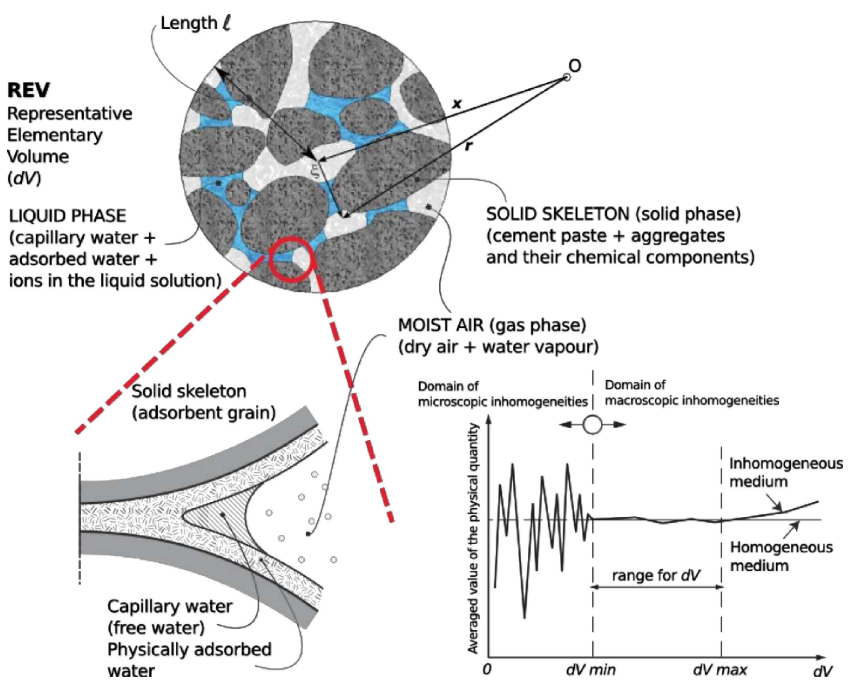

Fig. 1. Schematic representation of the moist concrete as a multiphase porous material

The thermodynamic quantity $\psi^{\kappa}$ to be introduced into Eq. (1) can be mass, momentum, angular momentum, energy or entropy. The relevant thermodynamic properties $\psi^{\kappa}$ for the different balance equations and values assumed by $\mathbf{i}_{\Psi}^{\kappa}$, $\mathbf{b}_{\Psi}^{\kappa}$ and $\mathbf{G}_{\Psi}^{\kappa}$ are listed in Table 1 .

Table 1

Thermodynamic properties for the microscopic mass balance equations

\begin{tabular}{ccccc}
\hline \hline Quantity & $\psi^{\kappa}$ & $\mathbf{i}_{\Psi}^{\kappa}$ & $\mathbf{b}_{\Psi}^{\kappa}$ & $\mathbf{G}_{\Psi}^{\kappa}$ \\
\hline Mass & 1 & 0 & 0 & 0 \\
\hline Momentum & $\dot{\mathbf{r}}$ & $\mathbf{t}_{m}^{\kappa}$ & $\mathbf{g}$ & 0 \\
\hline Energy & $E^{\kappa}+\dot{\mathbf{r}} \cdot \dot{\mathbf{r}} / 2$ & $\mathbf{t}_{m}^{\kappa} \dot{\mathbf{r}}-\mathbf{q}^{\kappa}$ & $\mathrm{g} \cdot \dot{\mathbf{r}}+h^{\kappa}$ & 0 \\
\hline Entropy & $\lambda^{\kappa}$ & $\boldsymbol{\Phi}^{\kappa}$ & $s^{\kappa}$ & $\varphi^{\kappa}$ \\
\hline
\end{tabular}

In Table $1, E^{\kappa}(\mathbf{r}, t)$ is the specific intrinsic energy, $\lambda^{\kappa}(\mathbf{r}, t)$ the specific entropy, $\mathbf{t}_{m}^{\kappa}(\mathbf{r}, t)$ the microscopic stress tensor, $\mathbf{q}^{\kappa}(\mathbf{r}, t)$ the heat flux vector, $\boldsymbol{\Phi}^{\kappa}(\mathbf{r}, t)$ the entropy flux, $\mathbf{g}$ the external momentum supply related to gravitational forces, $h^{\kappa}(\mathbf{r}, t)$ the intrinsic heat source, $s^{\kappa}(\mathbf{r}, t)$ the intrinsic entropy source and $\varphi^{\kappa}(\mathbf{r}, t)$ denotes the entropy increase. All the above mentioned quantities concern the $\kappa$-phase. The constituents are assumed to be microscopically non-polar, hence the angular momentum balance equation has been omitted here. This equation shows however that the stress tensor is symmetric.

2.2. Volume averaging procedure and macroscopic conservation equations. The final form of the macroscopic balance equations is obtained by applying appropriate space averaging operators (i.e. integrations applied for the so called Representative Elementary Volume - REV, Fig. 1) to Eq. (1) at micro-level, while the constitutive laws are defined directly at the upper scale, according to the so called Hybrid Mixture Theory (HMT) originally proposed by Hassanizadeh and Gray [20-22].

The dimensions of REV should be chosen in a proper way [18] (see Fig. 1), e.g. for concrete the REV radius should be approximately 3 times greater than the dimension of the largest aggregate. It is also possible to use of a numerical multi-scale approach (i.e. numerical averaging in REV) in the formulation of the material properties, which nowadays is often used for solving problems involving multi-physics aspects in material mechanics, see e.g. [23] for its application in analysis of internal curing of maturing concrete.

For the sake of brevity, only the final form of the macroscopic conservation equation (obtained from their integral form) is given below. All the physical quantities in these Eqs. are volume averaged, within the whole REV (denoted with subscript, e.g. $\langle\rho\rangle_{s}$ for apparent density of skeleton) or within the volume of $\kappa$-phase in REV only (denoted with superscript, e.g. $\langle\rho\rangle^{s}$ for real density of skeleton material). For details of the averaging procedure and its mathematical proofs the Reader can refer to $[18,20]$, and the kinematics of porous medium assumed at macro-level is described in [18].

In what follows the symbols of volume averaging, $\langle\cdot\rangle_{\kappa}$ and $\langle\cdot\rangle^{\kappa}$, are omitted, because all the physical quantities are volume averaged. The position of index indicates if the considered quantity is averaged within whole REV, e.g. $\rho_{s}$, or within the $\kappa$-phase in REV only, e.g. $\rho^{s}$. After the space averaging, i.e. at macroscopic level, porous medium is modelled as overlapping continua of the phases/components. This means that in every point of the material at macro-scale, $\mathbf{x}$, all the phases/components are present, and the fields $\psi^{\kappa}(\mathbf{x}, t)$ describing their properties are continuous in space. The full development of the model equations, starting from the local, microscopic balance Eqs. (1) with successive volume averaging, is presented in $[6,18,24]$.

The conservation equations at macroscopic level are written in terms of chosen primary variables: gas pressure, $p^{g}(\mathbf{x}, t)$, capillary pressure, $p^{c}(\mathbf{x}, t)\left(p^{c}=p^{g}-p^{w}\right.$, where $p^{w}(\mathbf{x}, t)$ is water pressure $)$, temperature, $T(\mathbf{x}, t)$, mass concentration of chemical species $x$ in water, $c_{x}(\mathbf{x}, t)$, and displacement vector, $\mathbf{u}(\mathbf{x}, t)$. It is assumed that a salt or chemical species $x$ might be dissolved in the pore solution. Solute influences the density and dynamic viscosity of the liquid phase [11]. The more salt is contained by the porous material, the more moisture is adsorbed by the porous material at the same relative humidity [11].

It is assumed that salt or chemical species $x$ might exist in two phases: dissolved in pore water and in solid phase (i.e. as precipitated salt crystals or as a component $x$ of the skeleton). Only the liquid form is mobile. Leaching of chemical species $x$ and salt crystallization/dissolution, as well as their influence on the properties of the skeleton and an additional pressure generated during the crystallization are considered. The kinetics of the chemical reaction is also taken into account, see e.g. [9, 12-15].

The macroscopic dry air mass balance equation takes into account both diffusive and advective air flow, as well as variations of porosity caused by chemical processes and deformations of the skeleton. It has the following form [18],

$$
\begin{gathered}
n \rho^{g a} \frac{D^{s} S_{g}}{D t}+S_{g} \rho^{g a} \frac{D^{s} n}{D t}+S_{g} n \frac{D^{s} \rho^{g a}}{D t} \\
+\operatorname{div} \mathbf{J}_{d}^{g a}+\operatorname{div}\left(n S_{g} \rho^{g a} \mathbf{v}^{g, s}\right)=0,
\end{gathered}
$$


where $\rho^{g a}(\mathbf{x}, t)$ is the dry air density, $\mathbf{J}_{d}^{g a}(\mathbf{x}, t)$ the dry air diffusive flux, $n(\mathbf{x}, t)$ the porosity, $S_{g}(\mathbf{x}, t)$ the saturation degree with gas, and $\mathbf{v}^{g, s}(\mathbf{x}, t)$ the velocity of gravity centre of the gas phase with respect to the skeleton.

The macroscopic liquid water balance considers advective flow of water (including osmotic flow of water in cement based materials, see [11]), mass sources related to phase changes of water (evaporation/condensation and physical adsorption/desorption), and variations of porosity caused by chemical processes and deformations of the skeleton, resulting in the following equation [18],

$$
\begin{gathered}
\rho^{w} n \frac{D^{s} S_{w}}{D t}+S_{w} n \frac{D^{s} \rho^{w}}{D t}+\rho^{w} S_{w} \frac{D^{s} n}{D t} \\
+\operatorname{div}\left(n S_{w} \rho^{w} \mathbf{v}^{w, s}\right)=-\dot{m}_{v a p},
\end{gathered}
$$

where $\rho^{w}(\mathbf{x}, t)$ is the liquid water density, $\dot{m}_{v a p}(\mathbf{x}, t)$ the mass source of gaseous water due to evaporation, $S_{w}(\mathbf{x}, t)$ the saturation degree with liquid water, and $\mathbf{v}^{w, s}(\mathbf{x}, t)$ the velocity of gravity centre of the pore water with respect to the skeleton.

The macroscopic mass balance equation of the solute $x$, dissolved in water, including dispersive and advective flow, mass sources related to chemical reactions, variations of porosity due to: chemical reactions, precipitation/dissolution of the solute $x$, and deformations of the skeleton, has the following form [12, 14, 15],

$$
\begin{gathered}
n S_{w} \frac{D^{s}\left(c_{x} \rho^{w}\right)}{D t}+n c_{x} \rho^{w} \frac{D^{s} S_{w}}{D t}+c_{x} S_{w} \rho^{w} \frac{D^{s} n}{D t}+\operatorname{div} \mathbf{J}_{D}^{x} \\
\quad+\operatorname{div}\left[n c_{x} S_{w} \rho^{w}\left(1-\sigma_{x}\right) \mathbf{v}^{w, s}\right]=\dot{m}_{x}+\dot{m}_{\text {leach }}^{x}
\end{gathered}
$$

where $\mathbf{J}_{D}^{x}(\mathbf{x}, t)$ is the dispersive flux of chemical species $x$, induced by the solute concentration gradient (including effect of osmosis for cement based materials [11]), $\sigma_{x}$ the so called reflection coefficient describing degree of semi-permeability of the material for the chemical species $x$ ( $\sigma_{x}=0$ for the 'no osmosis' case $), \dot{m}_{x}(\mathbf{x}, t)$ and $\dot{m}_{\text {leach }}^{x}(\mathbf{x}, t)$ the sources of the solute $x$ due to its dissolution from the $x$-crystals and due to leaching from the skeleton, respectively.

The energy balance equation (for the whole system) accounting for the conductive and convective heat flow, heat effects of phase changes, dissolution/precipitation processes, can be written as $[12,15]$,

$$
\begin{gathered}
\left(\rho C_{p}\right)_{e f} \frac{D^{s} T}{D t}+\left(\rho_{w} C_{p}^{w} \mathbf{v}^{w, s}+\rho_{g} C_{p}^{g} \mathbf{v}^{g, s}\right) \cdot \operatorname{grad} T \\
-\operatorname{div}\left(\chi_{e f} \operatorname{grad} T\right)=-\dot{m}_{\text {vap }} \Delta H_{\text {vap }}-\dot{m}_{x} \Delta H_{\text {prec }}^{x} \\
-\dot{m}_{\text {leach }}^{x} \Delta H_{\text {leach }}^{x}
\end{gathered}
$$

where $\left(\rho C_{p}\right)_{e f}=(1-n) \rho^{s} C_{p}^{s}+n S_{w} \rho^{w} C_{p}^{w}+n S_{g} \rho^{g} C_{p}^{g}+$ $n S_{p} \rho^{x} C_{p}^{x}$ means the effective (apparent) isobaric thermal capacity of the multiphase material, $C_{p}^{\kappa}$ the isobaric specific heat of $\kappa$-phase $(\kappa=s, w, g, x), \rho^{x}$ the density of precipitated chemical species $x, \chi_{e f}(\mathbf{x}, t)$ the effective thermal conductivity, while the enthalpies of vaporization, precipitation / dissolution and leaching are respectively:

$$
\begin{gathered}
\Delta H_{\text {vap }}=H^{g w}-H^{w}, \quad \Delta H_{p r e c}^{x}=H_{w}^{x}-H_{p}^{x}, \\
\Delta H_{\text {leach }}^{x}=H_{w}^{x}-H_{s}^{x} .
\end{gathered}
$$

The linear momentum balance equation for the multiphase system, in the rate form, with neglecting the inertial forces (only slow phenomena are considered here), is given by [5, 12, 18],

$$
\operatorname{div}\left(\dot{\mathbf{t}}^{\text {total }}\right)+\dot{\rho}_{e f} \mathbf{g}=0,
$$

where $\mathbf{t}^{\text {total }}(\mathbf{x}, t)=\sum_{\kappa} \mathbf{t}^{\kappa}(\mathbf{x}, t)$ is the total stress tensor, $\rho_{\text {ef }}(\mathbf{x}, t)=(1-n) \rho^{s}+n S_{w} \rho^{w}+n S_{g} \rho^{g}+n S_{p} \rho^{x}$ means the apparent density of the multiphase material, $\mathbf{g}$ the vector of gravity acceleration. The volume averaged angular momentum balance equation shows [18], that for non-polar media, as building materials are assumed in this work, all macroscopic partial and total stress tensors are symmetric, i.e. $\mathbf{t}^{\kappa}=\left(\mathbf{t}^{\kappa}\right)^{T}$ and $\mathbf{t}^{\text {total }}=\left(\mathbf{t}^{\text {total }}\right)^{T}$.

From the macroscopic entropy inequalities for the medium constituents, limitations for the form of constitutive relationships can be deduced, e.g. using the Colemann-Noll procedure, as done in [8]. This assures that the constitutive relations do not violate the Second Law of Thermodynamics.

To obtain the final form of mass balance Eqs. (2)-(4), the time derivative of porosity is eliminated from them using the macroscopic solid skeleton mass balance equation. It accounts for variations of porosity due to both the skeleton deformations and leaching process of the chemical species $x$ from the skeleton [9],

$$
(1-n) \frac{D^{s} \rho^{s}}{D t}-\rho^{s} \frac{D^{s} n}{D t}+\rho^{s}(1-n) \operatorname{divv}^{s}=-\dot{m}_{\text {leach }}^{x},
$$

where $\rho^{s}$ is the solid skeleton density.

Then, the mass source of water due to evaporation is eliminated from Eqs. (3) and (5) by means of the macroscopic vapour mass balance equations. This equation including diffusive and advective flow of vapour, mass sources related to the phase changes of water, and the variations of porosity due to chemical reactions and deformations of the skeleton, has the following form [18],

$$
\begin{gathered}
\rho^{g w} n \frac{D^{s} S_{g}}{D t}+S_{g} n \frac{D^{s} \rho^{g w}}{D t}+\rho^{g w} S_{g} \frac{D^{s} n}{D t}+\operatorname{div} \mathbf{J}_{d}^{g w} \\
+\operatorname{div}\left(n S_{g} \rho^{g w} \mathbf{v}^{g, s}\right)=\dot{m}_{v a p},
\end{gathered}
$$

where $\rho^{g w}(\mathbf{x}, t)$ is the water vapour density and $\mathbf{J}_{d}^{g w}(\mathbf{x}, t)$ the water vapour diffusive flux.

Finally, the mass source terms in Eqs. (4), (5) and (8), related to the chemical processes considered, are expressed by means of the appropriate evolution equations describing the processes' kinetics. They are dealt with in next subsection.

2.3. Evolution equations. In this subsection only calcium leaching from the material skeleton and salt precipitation/crystallization in the pore solution is described in detail. The progress of these chemical processes is given by the evolution equations written in terms of the internal variables: the calcium degree of leaching, $\Gamma_{\text {leach }}(\mathrm{x}, t)$, and the saturation degree with precipitated salt, $S_{p}(\mathrm{x}, t)$. The calcium leaching degree is defined in terms of the initial (i.e. for chemically not deteriorated, sound material) and the actual values of solid calcium content, which might be leached from the skeleton, $s_{C a}^{0}$ and $s_{C a}(\mathbf{x}, t)$, as follows [9]: 


$$
\Gamma_{\text {leach }}^{C a}(\mathbf{x}, t)=\frac{s_{C a}^{0}-s_{C a}(\mathbf{x}, t)}{s_{C a}^{0}} .
$$

Hence its value might increase from 0 to 1.0 during progressing leaching process.

The evolution equation for non-isothermal calcium leaching has the following form [9, 12],

$$
\frac{d \Gamma_{\text {leach }}^{C a}}{d t}=-\frac{A_{\text {leach }}^{C a}}{s_{\text {Ca }}^{0} R T_{\text {ref }} \tau_{\text {leach }}^{C a}},
$$

where chemical affinity of the calcium leaching process is defined as follows,

$$
A_{\text {leach }}^{C a}=\int_{s_{C a}^{0}}^{s_{C a}^{*}} \kappa\left(\bar{s}, T_{\text {ref }}\right) d \bar{s}-\int_{s_{C a}^{0}}^{s_{C a}} \kappa\left(\bar{s}, T_{\text {ref }}\right) d \bar{s} .
$$

$\tau_{\text {leach }}=f\left(s_{C a}, T\right)$ is the characteristic time of the process, $T_{\text {ref }}$ the reference temperature, $\kappa$ the equilibrium function, $s_{C a}^{0}$ and $s_{C a}(\mathbf{x}, t)$ are the initial (i.e. for sound material) and actual values of solid calcium content (i.e. the calcium which can be leached from the skeleton). The characteristic time $\tau_{\text {leach }}$ for different phases of solid calcium can be expressed by the following approximate relationships [12]:

$$
\tau_{\text {leach }}^{\pi}(T) \cong \tau_{\text {leach }}^{\pi}\left(T_{\text {ref }}\right) \times \exp \left[A_{\tau}\left(T-T_{\text {ref }}\right)\right],
$$

where $T_{\text {ref }}=298.15 \mathrm{~K}, A_{\tau}=-0.0239 \mathrm{~K}^{-1}$, and superscript $\pi=$ por, etr, $C S H$ means the portlandite, ettringite or $\mathrm{CSH}$ phases, respectively.

Equations $(11 \mathrm{a}, \mathrm{b})$ determine the rate of calcium leaching process. In order to calculate the degree of process advancement, one has to integrate Eq. (11) over the considered period of time, according to the equation:

$$
\Gamma_{\text {leach }}^{\text {Ca }}(\mathbf{x}, t)=\Gamma_{\text {leach }}^{\text {Ca }}\left(\mathbf{x}, t_{0}\right)+\int_{t_{0}}^{t} \dot{\Gamma}_{\text {leach }}^{\text {Ca }}(\mathbf{x}, t) d t .
$$

The mass source term, describing the calcium leaching in Eqs. (4), (5) and (8), is given by [12],

$$
\dot{m}_{\text {leach }}^{\text {Ca }}(\mathbf{x}, t)=M_{\text {leach }}^{C a} s_{C a}^{0} \cdot \dot{\Gamma}_{\text {leach }}^{C a}(\mathbf{x}, t),
$$

where $M_{\text {leach }}^{C a}$ is the average molar mass of dissolved calcium from the solid skeleton, assumed here as the average value for the portlandite, ettringite and CSH-pastes [12]. One should underline that non-isothermal calcium leaching is an irreversible process and it is described here by a non-equilibrium model, what allows to take into account the effect of variable temperature, moisture content and calcium ions concentration of the process progress rate. More details about this model can be found in [12].

The progress of salt crystallization/dissolution is described in terms of the pore saturation degree with precipitated salt, i.e. $S_{p}(\mathbf{x}, t)=V_{p}(\mathbf{x}, t) / V_{\text {pore }}(\mathbf{x}, t)$, which during the process might increase from 0 up to 1.0, when the whole pore volume is occupied by crystals of salt. Liquid water is assumed incompressible here and the model can be applied only below the limit value of $S_{p, \max }(\mathbf{x}, t)<1.0-S_{w}(\mathbf{x}, t)$, when serious numerical problems arise.
The evolution equation for salt precipitation/dissolution, depending on the solution supersaturation ratio, might be described by the non-equilibrium Freundlich isotherms [13, 14],

$$
\frac{d S_{p}}{d t}=\left\{\begin{array}{l}
S_{w}(t) H\left(S(t)-A^{\prime}\right)^{p}, \quad S \geq A^{\prime} \wedge S_{p}<1 \\
-S_{w}(t) H|S(t)-1|^{p}, \quad S<1 \wedge 0<S_{p}<1
\end{array}\right.
$$

where $p$ is the process order, $H$ the constant depending on the kind of salt and the properties of porous material.

The first line of Eq. (15) concerns the salt crystallization from the supersaturated solution, while the second line describes the salt crystals dissolution, which proceeds when the solution supersaturation ratio is lower than 1.0. $A^{\prime}$ is the primary crystallization coefficient, the value of which might vary from 1.7 up to 10 , depending on the kind of salt, pores structure and the solid composition [25, 26]. Equation (15) describes the evolution of salt crystals volume (i.e. salt precipitation process). It is assumed that there is no salt in the solid phase until the solution becomes supersaturated. Most salt solutions are the electrolytes so they decompose into ions (cations and anions), therefore the ion interaction must be considered while modelling their transport and crystallization. The salt crystallization is induced by the existence of nonequilibrium of the chemical potentials of salt in the solid- and liquid phase. Quantitatively this driving potential is described by the solution supersaturation ratio, $S^{x}(\mathbf{x}, t)=a_{x} / K_{x}$, where $a_{x}(\mathbf{x}, t)$ means the ion activity and $K_{x}$ the equilibrium constant for salt $x$.

The ion activity for hydrated salt $x$ is calculated according to the equation [27]:

$$
\begin{aligned}
\ln a_{x}=v_{M} & \ln \left(m_{M}\right)+v_{X} \ln \left(m_{X}\right)+v_{M} \ln \left(\gamma_{M}\right) \\
& +v_{X} \ln \left(\gamma_{X}\right)+v_{0} \ln \left(a_{w}\right),
\end{aligned}
$$

where $m_{M}(\mathbf{x}, t)$ and $m_{X}(\mathbf{x}, t)$ are the molalities of cations (described with subscript $M$ ) and anions $(X)$, respectively, $\gamma_{M}, \gamma_{X}$ the activity coefficients of the ions, $v_{M}, v_{X}, v_{0}$ the stoichiometric coefficients, and $a_{w}(\mathbf{x}, t)$ the water activity. In order to calculate the ion activity coefficients, the Pitzer ion interaction model is employed [28].

Equation (15) describes the evolution of salt crystals volume, determining the rate of crystals growth or dissolution. In order to calculate the total volume of salt crystals present in the pore system, one has to integrate Eq. (15) over the considered period of time, according to the equation:

$$
S_{p}(\mathbf{x}, t)=S_{p}\left(\mathbf{x}, t_{0}\right)+\int_{t_{0}}^{t} \dot{S}_{p}(\mathbf{x}, t) d t
$$

The mass source term in Eqs. (4) and (5), describing the salt precipitation in pore solution, is given by [13],

$$
\dot{m}_{x}(\mathbf{x}, t)=n \rho^{x} \dot{S}_{p}(\mathbf{x}, t) .
$$

\section{Effective stress principle for building materials exposed to chemical degradation}

Moist building materials are multi-phase porous media, hence analyzing the stress state and the deformation of the material 
it is necessary to consider not only the action of an external load, but also the pressure exerted on the skeleton by fluids and crystals present in its voids. The total stress tensor acting in a point of the porous medium may be split into the effective stress $\mathbf{t}^{e f}(\mathbf{x}, t)$, which accounts for stress effects due to changes in porosity, spatial variation of porosity and the deformations of the solid matrix, and a part accounting for the solid phase pressure exerted by the fluids and crystals present in pore volume, $p^{s}(\mathbf{x}, t),[7,29,30]$,

$$
\mathbf{t}^{t o t}=\mathbf{t}^{e f}-p^{s} \mathbf{I}=\mathbf{t}^{e f}-\left(p^{g}-\chi_{s}^{w s} p^{c}-\chi_{s}^{c s} p^{c r y s t}\right) \mathbf{I},
$$

where $\mathbf{I}$ is the second order unit tensor, $p^{\text {cryst }}(\mathbf{x}, t)$ the crystallization pressure, while $\chi_{s}^{w s}\left(S_{w}\right)$ and $\chi_{s}^{c s}\left(S_{p}\right)$ the fractions of skeleton area in contact with liquid water and salt crystals, respectively.

In Eq. (19) capillary pressure, $p^{c}(\mathbf{x}, t)$, is defined in such a way that it has physical meaning also in the hygroscopic range of moisture content, [29],

$$
p^{c} \equiv p^{g}-p^{w}=\Pi^{f}-\gamma^{w g} J_{w g}^{w} .
$$

In this region it takes also into account the effect of disjoining pressure, $\Pi^{f}(\mathbf{x}, t)$, besides the curvature and surface tension of the water/gas interface, $J_{w g}^{w}\left(S_{w}, T\right)$ and $\gamma^{w g}(\omega, T)$. At higher relative humidity, in the capillary moisture range, the effect of disjoining pressure is neglected $\left(\Pi^{f}=0\right)$.

The precipitated salt crystals are considered as an additional (with respect to the skeleton) solid phase, which does not participate in the transmission of external load. The growing salt crystals exert an additional crystallization pressure, $p^{\text {cryst }}(\mathbf{x}, t)$, on the solid skeleton which acts as an obstacle confining crystals between the pore walls [31]. The crystals exert the pressure on the material skeleton only if a thin solution film is situated between the growing crystal and the skeleton. In order to describe the crystallization pressure, the chemical potentials on the loaded and unloaded faces of the crystal and the chemical potential of the solute are compared. The crystallization pressure $p^{\text {cryst }}(\mathbf{x}, t)$ is given by the following formula [27]:

$$
p^{\text {cryst }}=\frac{R T}{V_{m}^{x}} \ln \left(\frac{a_{x}}{K_{x}}\right),
$$

where $R$ is the universal gas constant and $V_{m}^{x}$ is the molar volume of salt $x$.

In order to take into account the effects of micro-cracking processes due to chemical degradation of the material strength properties, caused by the leaching process or the salt crystallization, a scalar damage model has been adopted here, similarly to what done in $[6,8]$. The use of a separate measure of damage for the chemical deterioration process $(V)$ potentially allows for a clear distinction between such a process and the evolution of damage of different nature (i.e. mechanical cracking).

The chemical damage variable $V(\mathbf{x}, t)$ for the leaching of $x$-species is defined as [12],

$$
V(\mathbf{x}, t)=1-\frac{E\left(\Gamma_{\text {leach }, \max }^{x}(\mathbf{x}, t)\right)}{E_{0}}
$$

and for the salt crystallization as,

$$
V(\mathbf{x}, t)=1-\frac{E\left(S_{p, \max }(\mathbf{x}, t)\right)}{E_{0}},
$$

where $E_{0}$ and $E$ are the Young moduli of a sound and chemically deteriorated material, respectively, and $S_{p, \max }(\mathbf{x}, t)$ is the highest value of pore saturation degree with precipitated salt, reached in point $\mathbf{x}$ until time instant $t$. This means that the chemical degradation of building materials is irreversible, though the salt precipitation itself is reversible. If leaching process of $x$-species is reversible, the maximal value of leaching degree reached until time instant $t, \Gamma_{\text {leach,max }}^{x}$, should be substituted by its actual value, $\Gamma_{\text {leach }}^{x}$. For example calcium leaching from cementitious building materials is an irreversible chemical process, hence the value of $\Gamma_{\text {leach, } \max }^{C a}$ must be used in Eq. (22). The dependence of the material Young modulus on the advancement of the chemical degradation process, i.e. $E\left(\Gamma_{\text {leach }}^{x}(\mathbf{x}, t)\right)$ or $E\left(S_{p}(\mathbf{x}, t)\right)$ should be determined experimentally. For calcium leaching in cementitious materials, a linear dependence of the material Young modulus $E\left(\Gamma_{\text {leach }}^{C a}(\mathbf{x}, t)\right)$ on the leaching degree $\Gamma_{\text {leach }}^{C a}$ can be used as a good approximation [12], hence chemical damage (22) is a linearly decreasing function of $\Gamma_{\text {leach }}^{C a}$ (and hence calcium content $s_{C a}$, see Eq. (10)).

Considering that in this work building materials are treated as multiphase porous media, also for the chemical deterioration processes the effective stress tensor $\mathbf{t}^{e f}(\mathbf{x}, t)$ has been used in the constitutive relationship [12],

$$
\mathbf{t}^{e f}=(1-V) \mathbf{D}_{0}\left(\varepsilon_{t o t}-\varepsilon_{c}-\varepsilon_{c h}-\varepsilon_{t}\right),
$$

where $\varepsilon_{t o t}(\mathbf{x}, t), \varepsilon_{c}(\mathbf{x}, t), \varepsilon_{c h}(\mathbf{x}, t)$ and $\varepsilon_{t}(\mathbf{x}, t)$ are the total-, creep-, chemical- and thermal strain tensors, and $\mathbf{D}_{0}(\mathbf{x}, t)$ is the tangent matrix for the material which is not chemically deteriorated. Shrinkage and creep strains are modelled by means of the effective stresses [7].

\section{Governing equations of the mathematical model and their numerical solution}

For closure of the mathematical model describing coupled hydro-thermo-chemo-mechanical phenomena in porous building materials, which consists of the mass-, energy- and momentum balance Eqs. (2)-(5) and (7), several constitutive and physical relationships, as well as the initial and boundary conditions, are needed. Due to lack of space, they are not recalled here. The complete set of these equations, describing hydro-thermal properties of porous building materials, can be found in $[18,24]$. The mechanical properties, necessary for modelling stresses and strains of building materials, including creep and cracking of cement based materials, are dealt with in $[7,8,30]$. The relationships needed for analysis of salt transport (including osmosis in cement based materials) and precipitation in building materials are given in $[11,14$, 15], and those necessary for modelling of calcium leaching are described in detail in [12].

After introducing all the aforementioned constitutive and physical relationships, initial and boundary conditions, as well as application of Galerkin's method with discretization of the 
state variables in space by means of finite element method $[18,32]$,

$$
\begin{aligned}
p^{g}(\mathbf{x}, t) & =\mathbf{N}_{p}(\mathbf{x}) \overline{\mathbf{p}}^{g}(t), \\
p^{c}(\mathbf{x}, t) & =\mathbf{N}_{p}(\mathbf{x}) \overline{\mathbf{p}}^{c}(t), \\
T(\mathbf{r}, t) & =\mathbf{N}_{t}(\mathbf{x}) \overline{\mathbf{T}}(t), \\
c_{x}(\mathbf{x}, t) & =\mathbf{N}_{s}(\mathbf{x}) \overline{\mathbf{c}}_{x}(t), \\
\mathbf{u}(\mathbf{x}, t) & =\mathbf{N}_{u}(\mathbf{x}) \overline{\mathbf{u}}(t),
\end{aligned}
$$

where $\overline{\mathbf{p}}^{g}(t), \overline{\mathbf{p}}^{c}(t), \overline{\mathbf{T}}(t), \overline{\mathbf{c}}_{x}(t), \overline{\mathbf{u}}(t)$ are the vectors of nodal values of the state variables, $\mathbf{x}$ the position vector, and $\mathbf{N}_{\kappa}(\mathbf{x})$ the shape functions of the state variables, the following matrix form of the governing equations is obtained [12],

$$
\mathbf{C}(\mathbf{X}) \frac{\partial \mathbf{X}}{\partial t}+\mathbf{K}(\mathbf{X}) \mathbf{X}=\mathbf{f}(\mathbf{X})
$$

with

$$
\begin{gathered}
\mathbf{C}=\left[\begin{array}{ccccc}
\mathbf{C}_{g g} & \mathbf{C}_{g c} & \mathbf{C}_{g t} & \mathbf{C}_{g s} & \mathbf{C}_{g u} \\
\mathbf{0} & \mathbf{C}_{c c} & \mathbf{C}_{c t} & \mathbf{C}_{c s} & \mathbf{C}_{c u} \\
\mathbf{0} & \mathbf{C}_{t c} & \mathbf{C}_{t t} & \mathbf{C}_{t s} & \mathbf{C}_{t u} \\
\mathbf{0} & \mathbf{C}_{s c} & \mathbf{C}_{s t} & \mathbf{C}_{s s} & \mathbf{C}_{s u} \\
\mathbf{C}_{u g} & \mathbf{C}_{u c} & \mathbf{C}_{u t} & \mathbf{C}_{u s} & \mathbf{C}_{u u}
\end{array}\right], \\
\mathbf{K}=\left[\begin{array}{ccccc}
\mathbf{K}_{g g} & \mathbf{K}_{g c} & \mathbf{K}_{g t} & \mathbf{K}_{g s} & \mathbf{0} \\
\mathbf{K}_{c g} & \mathbf{K}_{c c} & \mathbf{K}_{c t} & \mathbf{K}_{c s} & \mathbf{0} \\
\mathbf{K}_{t g} & \mathbf{K}_{t c} & \mathbf{K}_{t t} & \mathbf{0} & \mathbf{0} \\
\mathbf{K}_{s g} & \mathbf{K}_{s c} & \mathbf{K}_{s t} & \mathbf{K}_{s s} & \mathbf{0} \\
\mathbf{0} & \mathbf{0} & \mathbf{0} & \mathbf{0} & \mathbf{0}
\end{array}\right], \\
\mathbf{f}=\left\{\begin{array}{c}
\mathbf{f}_{g} \\
\mathbf{f}_{c} \\
\mathbf{f}_{t} \\
\mathbf{f}_{s} \\
\mathbf{f}_{u}
\end{array}\right\}, \\
\end{gathered}
$$

where the non-linear matrix coefficients $\mathbf{C}(\mathbf{X}), \mathbf{K}(\mathbf{X})$ and $\mathbf{f}(\mathbf{X})$ for the leaching problem are defined in detail in [12], and for salt crystallization in [33]. The integrals appearing in these matrices are calculated by means of the Gauss method [32]. To determine the values and time derivatives of the saturation degree with precipitated salt $S_{p}$ and/or of the leaching degree $\Gamma_{\text {leach }}^{x}$ in all Gauss' points an implicit Euler method (i.e. backward Finite Difference scheme [32]) is used,

$$
\begin{gathered}
\left(\dot{S}_{p}\right)_{n+1} \cong \frac{\left(\Delta S_{p}\right)_{n+1}}{\Delta t}=\frac{\left(S_{p}\right)_{n+1}-\left(S_{p}\right)_{n}}{\Delta t} \\
\left(\dot{\Gamma}_{\text {leach }}^{x}\right)_{n+1} \cong \frac{\left(\Gamma_{\text {leach }}^{x}\right)_{n+1}-\left(\Gamma_{\text {leach }}^{x}\right)_{n}}{\Delta t},
\end{gathered}
$$

where subscript $n$ means the time step number and $\Delta t$ is the time step length.

As can be observed in the equation set (26), the present mathematical model considers several mutual couplings between the hygral-, thermal-, chemical- and mechanical phenomena in porous building materials.

The time discretization of the equation set (26) is carried out by means of a fully implicit Finite Difference scheme (i.e. backward difference) [32],

$$
\begin{aligned}
& \Psi\left(\mathbf{X}_{n+1}\right)=\mathbf{C}\left(\mathbf{X}_{n+1}\right) \frac{\mathbf{X}_{n+1}-\mathbf{X}_{n}}{\Delta t} \\
& +\mathbf{K}\left(\mathbf{X}_{n+1}\right) \mathbf{X}_{n+1}-\mathbf{f}\left(\mathbf{X}_{n+1}\right)=\mathbf{0} .
\end{aligned}
$$

The nonlinear equation set (30) is solved by means of a monolithic Newton-Raphson type iterative procedure [18, 24],

$$
\begin{gathered}
\Psi\left(\mathbf{X}_{n+1}^{k}\right)=-\left.\frac{\partial \Psi}{\partial \mathbf{X}}\right|_{\mathbf{X}_{n+1}^{k}} \Delta \mathbf{X}_{n+1}^{k} \\
\mathbf{X}_{n+1}^{k+1}=\mathbf{X}_{n+1}^{k}+\Delta \mathbf{X}_{n+1}^{k}
\end{gathered}
$$

where $k$ is the iteration index and $\frac{\partial \Psi}{\partial \mathbf{X}}$ the Jacobian matrix.

Based on the presented mathematical model and methods for its numerical solution, the computer codes HMTRA-SALT [11, 13-15], and COMES-LEACH [9, 10, 12] have been developed by the Authors. All numerical examples presented in the next section have been solved by means of the software.

\section{Numerical examples}

Three numerical examples are solved in this section to demonstrate possibilities of the mathematical model presented in the previous sections. The first one, being an experimental validation of the mathematical model, deals with capillary suction of the salt solution by two building materials, the sandstone and cement mortar. The comparison of the simulation results with the experimental data shows, that due to very fine pore structure, osmotic phenomena are of importance in the cement mortar. The second numerical example concerns salt crystallization in a drying wall made of ceramic brick. The third example deals with calcium leaching from the concrete wall exposed to the gradient of water pressure at two different temperatures, the solution of which was previously not possible with the equilibrium type mathematical models of leaching.

5.1. Capillary suction of a salt solution by two building materials - mathematical model validation. To validate the proposed mathematical model and developed computer code HMTRA_SALT, two capillary suction experiments have been simulated and the numerical results have been compared with the experimental data. The first experiment, concerning a sample made of a sandstone, has been performed by Rucker et al. [34]. The sample was initially dry and then put into the saturated solution of $\mathrm{NaCl}$ in water. The solution was sucked into pores due to capillary forces, causing upwards transport of the solution. The salt and water content in the sample were measured by means of nuclear magnetic resonance (NMR) and gamma ray absorption. By combination of the two nondestructive techniques, both the moisture and salt profile have been determined.

Sandstone is a material with prevailing macro-pores, hence osmosis phenomenon is negligible in this case and the reflection coefficient $\sigma_{\mathrm{NaCl}}=0$ was assumed. The following material parameters were used in the simulations: density $\rho^{s}=2120 \mathrm{~kg} / \mathrm{m}^{3}$, porosity $n=17 \%$, intrinsic permeability $k=1.5 \cdot 10^{-15} \mathrm{~m}^{2}$, salt diffusion coefficient $D^{N a C l}=10^{-9} \mathrm{~m}^{2} / \mathrm{s}$. The simulations were performed for the first $46 \mathrm{~h}$ of the analyzed process. 
The comparison of the simulation results, concerning the changes of the water content and the salt concentration, with experimental data is presented in Fig. 2. Figure 2a shows water content and Fig. 2b salt content profiles after 8, 22, 32, 46 hours from the experiment beginning. As it can be seen the calculated results are in a good agreement with the experimental data.

a)

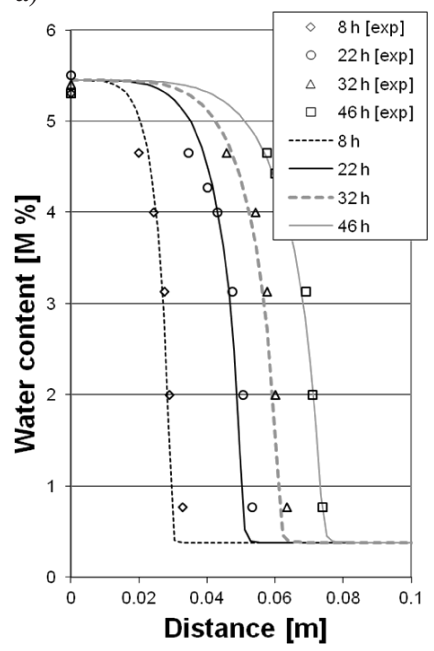

b)

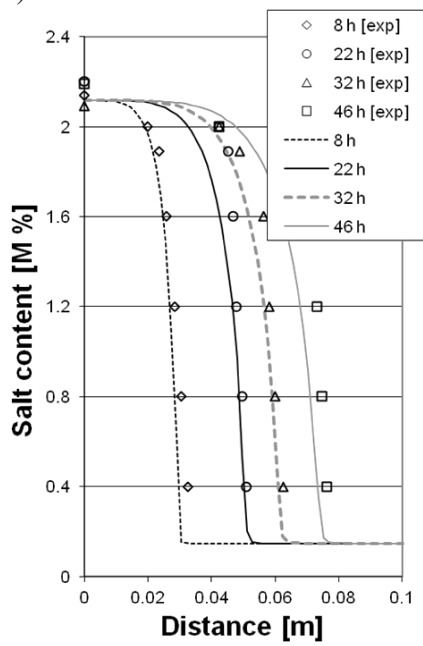

Fig. 2. Comparison of the simulation results with the experimental data of Rucker et al. [34] for $t=8,22,32$ and 46 hours after the start of capillary suction of the $\mathrm{NaCl}$ water solution by the sandstone: a) the water content, b) the salt content

The second experiment, concerning capillary suction of the $\mathrm{NaCl}$ water solution by a sample made of cement mortar (CEM I 42.5 R Lafarge), was performed by Cerny et al. [35]. To investigate the moisture and salt content profiles, the combination of gravimetric method and chemical analysis has been applied. Gel pores take a considerable part of pore volume of cement based materials [36] and the mean pore diameter of the cement mortar is about ten times smaller than that of the sandstone. Gel pores behave similarly as a semi-permeable membrane, which blocks salt transport and in the same time allow water to flow to the regions with higher salt concentration. This is the physical reason of osmotic flow of water in cement based materials, which is mathematically described by the reflection coefficient $\sigma_{\mathrm{NaCl}}$. In the presented simulations the reflection coefficient $\sigma_{\mathrm{NaCl}}=0.4$, determined in [11], was assumed. The simulations were performed for the first 7 days of the analyzed process.

Figures $3 \mathrm{a}$ and $3 \mathrm{~b}$ present the comparison of experimental data and computed results concerning the moisture and salt contents. As it can be noticed, the front of moisture content (Fig. 3a) moves faster than the front of salt content (Fig. 3b), what was caused by osmotic water flow due the presence of gel pores in the material micro-structure. Besides the capillary flow of water in the direction of decreasing water content, an additional flux of water particles tends to dilute the salt solution, so it is directed towards the higher salt concentration regions. In the analysed case this flux slows down the water transport. This example indicates that for some building materials with very fine micro-structure of porosity, like for example cement based materials, the osmosis should be taken into account during analyzing the mass transport processes. a)

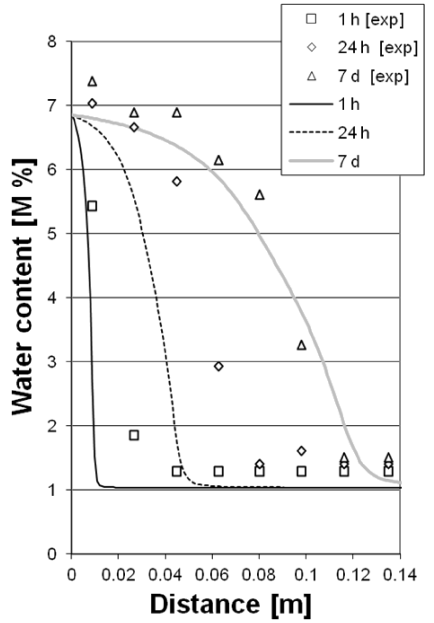

b)

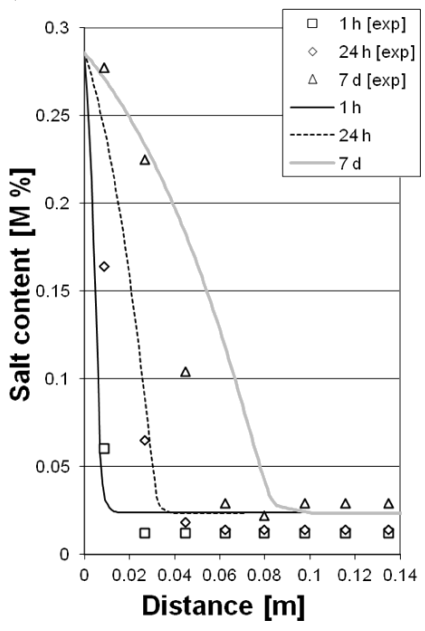

Fig. 3. Comparison of the simulation results with the experimental data of Cerny et al. after Ref. 35 for $t=1,7$ hours and 7 days after the start of the of capillary suction of the $\mathrm{NaCl}$ water solution by the cement mortar: a) water content, b) salt content

5.2. Salt crystalization in a drying brick wall. The two-side drying of a brick wall, contaminated with the $\mathrm{NaCl}$ solution, was simulated using the HMTRA_SALT software. The following material properties were assumed during the simulation: intrinsic permeability coefficient $k=3 \cdot 10^{-17} \mathrm{~m}^{2}$, porosity $n=0.293$, skeleton density $\rho^{s}=1834 \mathrm{~kg} / \mathrm{m}^{3}$. The process order constant in Eq. (15) was assumed as $p=7.5$, which value was determined in [33]. The sorption isotherm of the brick was measured by means of the saturated salt solution method, and it may be described by the following equations, proposed by Baroquel-Bouny et al. [37],

$$
p^{c}=a\left(S_{w}^{-b}-1\right)^{1-1 / b},
$$

with $a=5.63$ and $b=1.5$, [33].

On the both surfaces of the wall the external conditions were the same. Due to this symmetry, only one half of the wall was modelled with 100 isoparametric eight-nodes finite elements of equal size. The finite element mesh and the scheme of boundary condition are presented in Fig. 4. The wall drying was induced by a rapid decrease of relative humidity of the surrounding air at side $\mathrm{A}$ (Fig. 4) from $\mathrm{RH}_{0}=100 \%$ to $\mathrm{RH}_{\infty}=85 \%$, while the temperature was equal to $20^{\circ} \mathrm{C}$. Convective heat and moisture exchange was assumed at the surface with heat and mass surface coefficients: $\alpha_{c}=8 \mathrm{~W} / \mathrm{m}^{2} \mathrm{~K}$ and $\beta_{c}=0.008 \mathrm{~m} / \mathrm{s}$. On the all other sides of the domain (sides: B, C and D in Fig. 4) the zero mass flows and the zero heat fluxes were assumed. The simulations were performed for the time period of $500 \mathrm{~h}$. 
$\mathrm{C}$

D

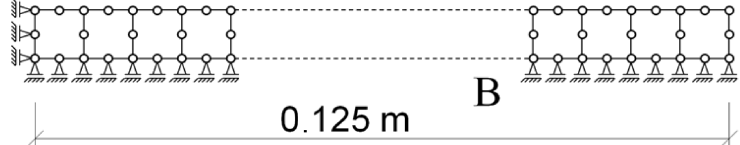

Fig. 4. Geometry and boundary condition of the analyzed example

The decrease of ambient air relative humidity causes the evaporation of water and the transport of the liquid phase from the interior of the wall toward its surface. Salt, migrating with water toward the surface, cannot evaporate, therefore its concentration gradually increases in the vicinity of the wall surface, where the solution often becomes supersaturated. Significant gradients of the supersaturation ratio are observed in the vicinity of the wall surface, Fig. 5a. It causes the salt diffusion in the opposite direction to the capillary flow. The diffusive flow tends to diminish the supersaturation gradient. When the solution is supersaturated, the salt crys- tallization proceeds. According to the simulation results, after $500 \mathrm{~h}$ of drying, $26 \%$ of the pore volume in the surface zone is occupied by the salt crystals, Fig. 5b. The growing crystals exert an additional pressure on the solid skeleton, which acts as an obstacle opposing the crystals formation, Fig. 5c. The crystallization pressure depends on the solution supersaturation ratio, see Eq. (21). Only a part of the solid internal surface is covered by the solid-crystal interface, which transmits the crystallization pressure. The crystallization pressure causes an additional tensile stresses in the solid skeleton. One can observe that during the salt crystallization positive tensile stresses are induced in the zone close to the surface, Fig. 5d. The tensile strength of the brick equals approximately $1 \mathrm{MPa}$. The results of simulation show that such value can be reached during the drying of brick contaminated with a salt. As a consequence, the brick can be destroyed due to the crystallization pressure, giving, as a brittle material, no signal of damage until the scaling appears.

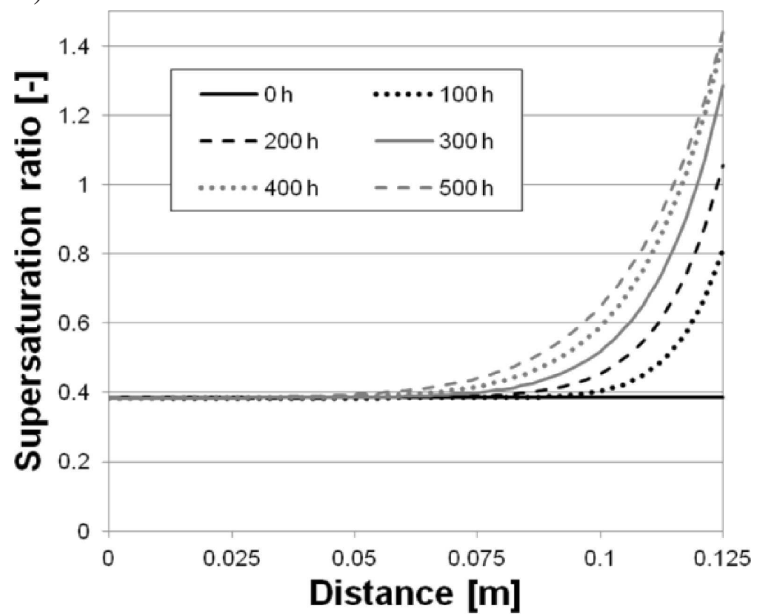

c)

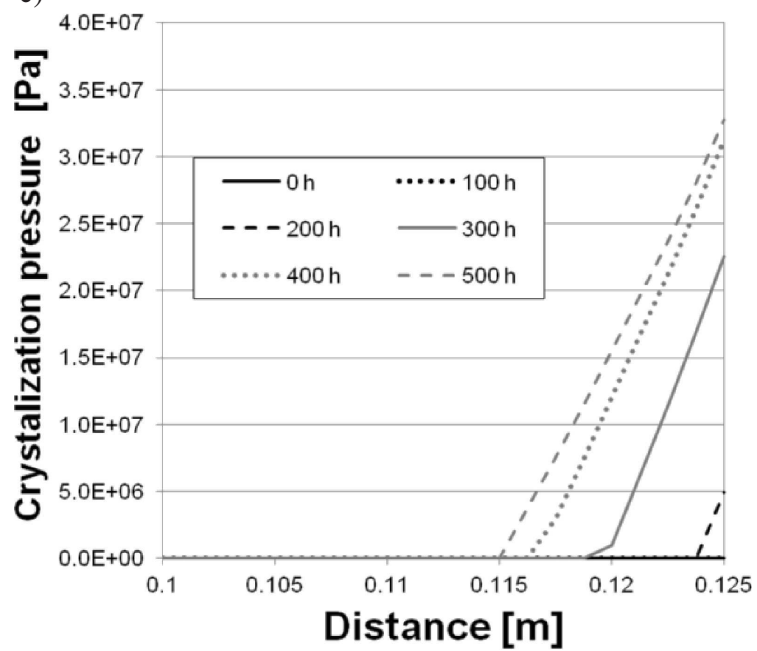

b)

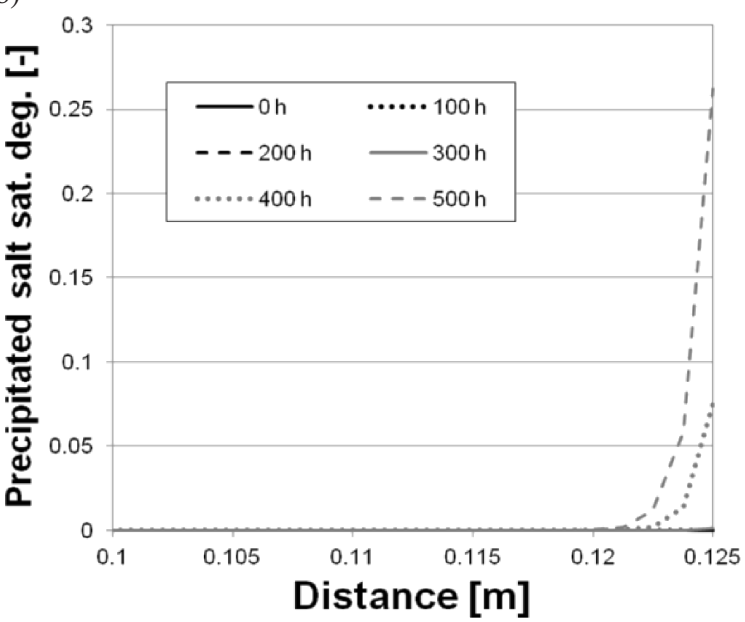

d)

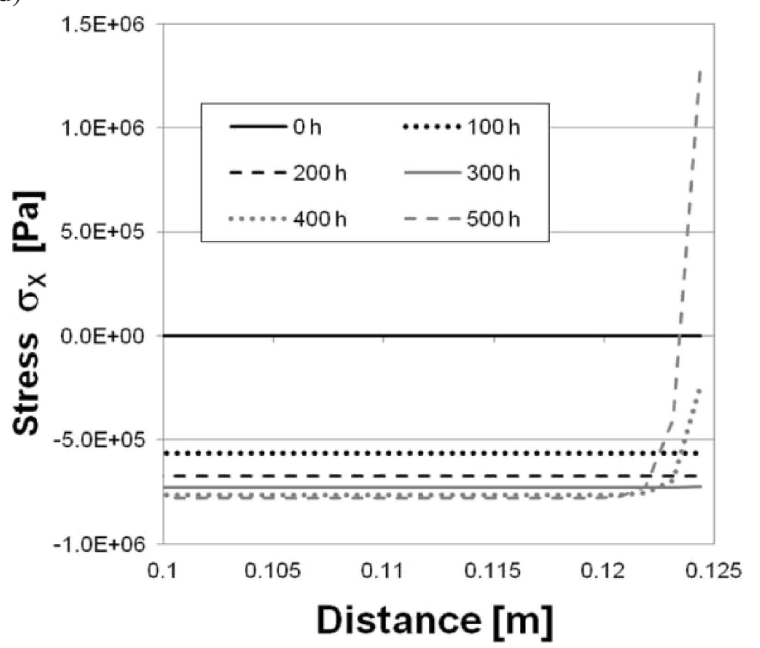

Fig. 5. The simulation results concerning drying of a brick contaminated with $\mathrm{NaCl}$ - profiles of state variables at different time stations: $t=100,200,300,400$ and $500 \mathrm{~h}$ : a) salt supersaturation ratio, b) relative volume of salt crystals, c) crystallization pressure in the surface zone, d) distribution of stresses in the surface zone 
5.3. Calcium leaching from a concrete wall exposed to water pressure gradient. The isothermal calcium leaching process (so called reaction-diffusion-advection problem), taking place in a concrete wall subjected to a water pressure difference of 5 bar between its two sides was numerically simulated using the COMES_LEACH software. The problem was solved for two different temperatures, $T=25^{\circ} \mathrm{C}$ and $T=60^{\circ} \mathrm{C}$. The pressure gradient leads to a deionized water inflow into the wall, see Fig. 6. There are no boundary conditions directly imposed for the exchange of calcium between the concrete element and outflowing water, but the calcium ions flux is the result of the water flux outflowing from the wall and the surface concentration of calcium. The following material properties were assumed during the simulation: intrinsic permeability coefficient $k=5 \cdot 10^{-19} \mathrm{~m}^{2}$, porosity $n=0.122$, Young modulus $E_{0}=30 \mathrm{GPa}$, Poisson's ratio $\nu=0.18$, compressive strength $f_{c}=34.5 \mathrm{MPa}$.

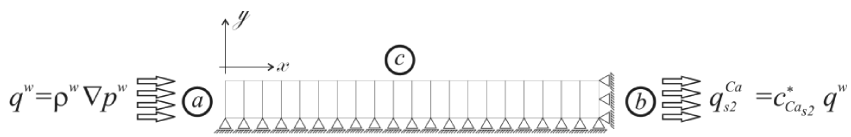

$$
\begin{aligned}
& T_{s l}, p_{s 1}^{w}=6 \mathrm{bar} \underset{\ell=16 \mathrm{~cm}}{\stackrel{(d)}{T_{s 2}}, p_{s 2}^{w}}=1 \mathrm{bar}
\end{aligned}
$$

Fig. 6. Geometry and boundary condition of the analyzed example

The wall was modelled by means of 100 isoparametric eight-nodes finite elements of equal size. The simulations are performed with constant time step length $\Delta t=0.1$ day, for the time span of $2 \times 10^{5}$ days.

The simulations were performed to assess the effect of temperature on the reaction-diffusion-advection process. Figures $7 \mathrm{a}$ and $7 \mathrm{~b}$ show the simulation results in terms of $\mathrm{Ca}^{2+}$ ions concentration in the liquid solution and calcium content in the solid skeleton at two different temperatures. In Fig. 7a it is possible to notice an increase of calcium concentration, with respect to the initial value, on the side of the wall where water flows out. The increase is higher for the temperature $T=25^{\circ} \mathrm{C}$. This phenomenon is caused by the advection processes carrying dissolved calcium from the left side, where deionized water flows into the concrete wall, to the right one. The temperature related difference in terms of calcium content in the solid skeleton is very distinct and increasing with the process progress, that is confirmed also from the evolution of chemical damage and the progress of the portlandite dissolution front (assumed as corresponding to $s_{C a} \cong 9 \mathrm{kmol} / \mathrm{m}^{3}$ ) in time, Figs. 8 and 9. The velocity of the front penetration (i.e. the slope of the straight lines in Fig. 9) is visibly higher for the temperature of $60^{\circ} \mathrm{C}$ than for $T=25^{\circ} \mathrm{C}$, what is also known from the practice and laboratory experiments.

One should underline that for the advection-diffusionreaction problem, the degradation front penetration is proportional to the time value and not to the square root of time, as in the case of purely diffusion-reaction problem, see e.g. [10, 12]. The results clearly show the importance of the temperature and the advection phenomenon for a correct simulation of the real behaviour of a concrete structure exposed to in- creased temperatures (as e.g. nuclear waste disposals) with regard to the calcium leaching process.

a)

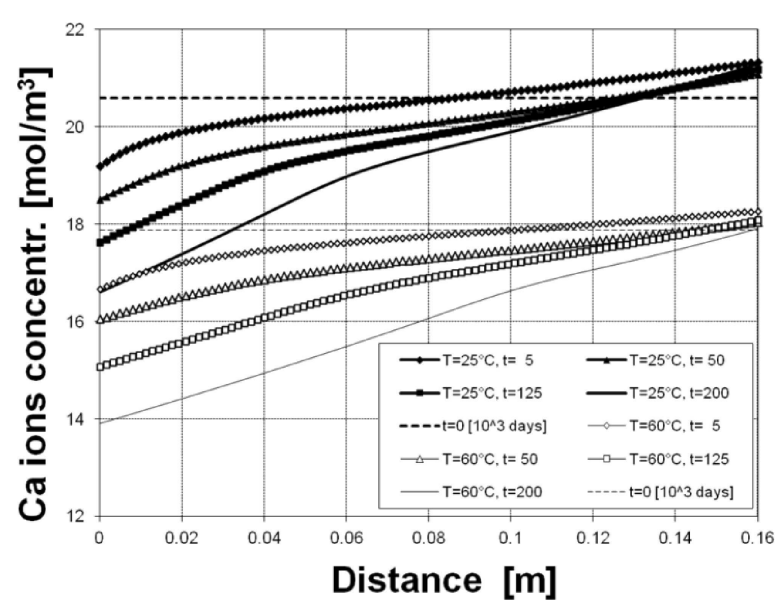

b)

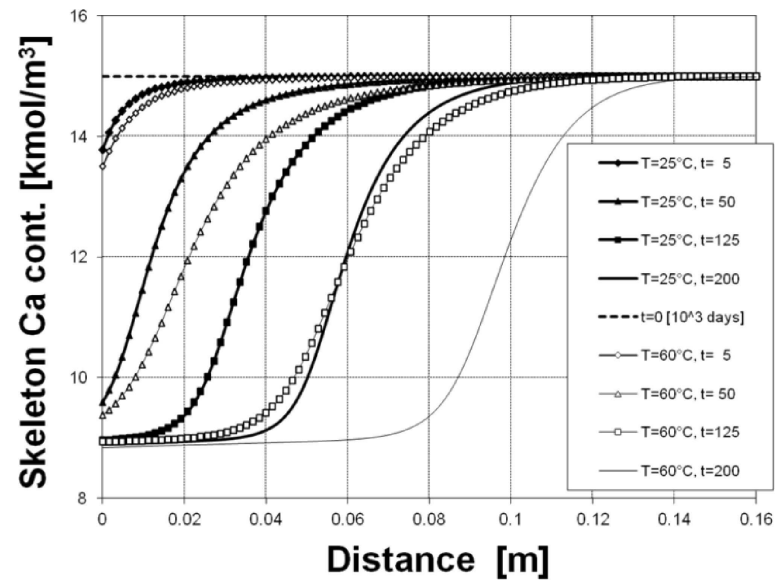

Fig. 7. Comparison of the space distributions of liquid calcium concentration (a) and solid calcium content (b) at four different time stations, obtained from simulations of the reaction-advectiondiffusion problem for the two different temperatures: $T=25^{\circ} \mathrm{C}$ and

$$
T=60^{\circ} \mathrm{C}
$$

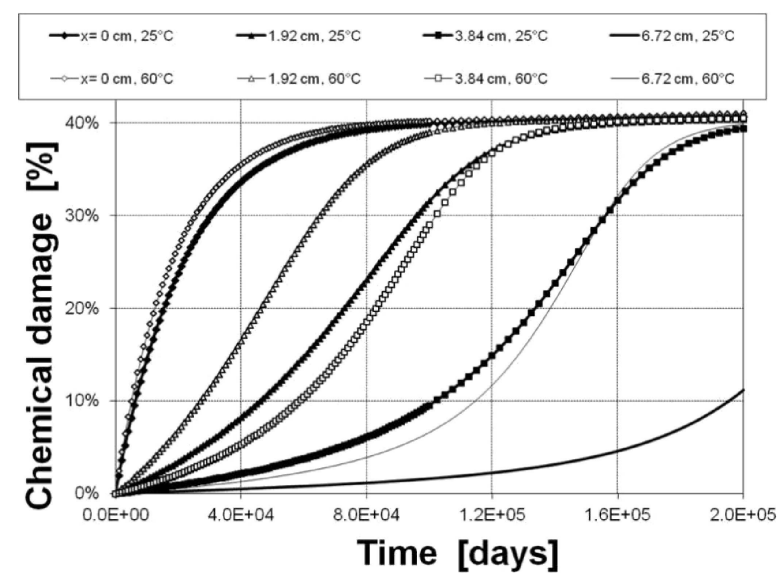

Fig. 8. Comparison of the time histories of chemical damage at four different positions (a) and the results concerning progress of the portlandite dissolution front in function of time, obtained from the simulations of the reaction-advection-diffusion problem for the two cases of temperature: $T=25^{\circ} \mathrm{C}$ and $T=60^{\circ} \mathrm{C}$ 


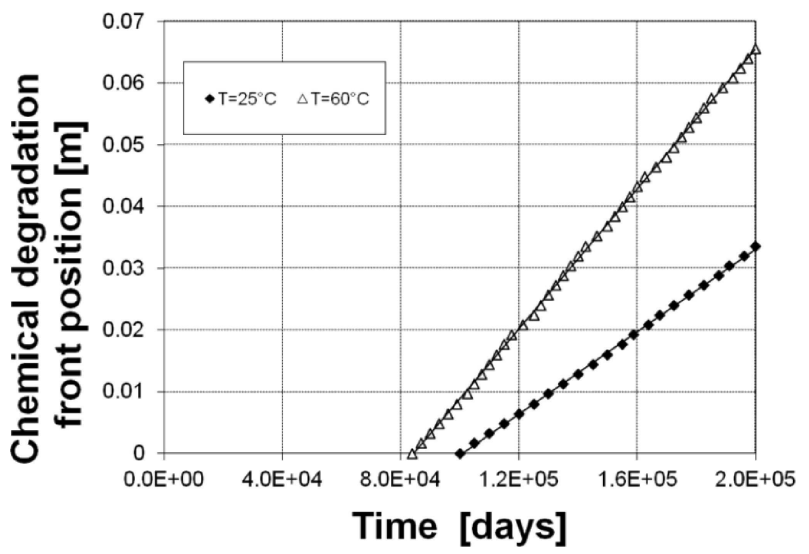

Fig. 9. Comparison of the results concerning progress of the portlandite dissolution front in function of time, obtained for the simulations of the reaction-advection-diffusion problem for the two cases of temperature: $T=25^{\circ} \mathrm{C}$ and $T=60^{\circ} \mathrm{C}$

\section{Conclusions}

A general mathematical model of hydro- thermo- chemo- mechanical behaviour of building materials, considering their multiphase nature and several mutual couplings of different physicochemical phenomena, has been briefly presented and numerically solved. Mechanics of multiphase porous media and damage mechanics are applied for this purpose, and kinetics of deterioration processes is described with evolution equations based on thermodynamics of chemical reactions, which allows for analysing building material degradation in variable environmental conditions (hydro-thermal and chemical). This approach can be adapted to very different types of chemical degradation, which are rather difficult for modelling, like calcium leaching, salt precipitation or other expansive chemical reactions, e.g. alkali silica reaction. The results of simulations performed show, that the mathematical model gives results which are in a good agreement with the present knowledge and few available experimental data concerning physicochemical processes leading to deterioration of building materials. Previous applications of the method to other difficult practical problems of civil engineering, e.g. concrete at high temperature, concrete at early ages, allowed for better understanding of their physics and explanation of some phenomena known from practice, like thermal spalling or autogeneous creep. Since the method gives clear definitions of all physical quantities involved, it allows for introducing constitutive relations at macroscopic level, using directly the results of experimental tests and/or numerical simulations at mesoscopic level of material structure. The model is based on physics of porous materials and widely uses the mechanistic approach, hence despite numerous model parameters, they are relatively easy for determination during simple laboratory tests. The main limitation of the approach is that it needs relatively fine FE mesh and short time steps, hence it is computationally costly and for this reason it is rather suitable for analyses at the material and element level rather than at the whole structure level.
Acknowledgements. The research has been partially funded by the project POIG.01.01.02-10-106/09-00 "Innovative means and effective methods to improve the safety and durability of building structures and transportation infrastructures in the strategy of sustainable development" at the Lodz University of Technology, Poland, within the "Innovative Economy Strategic Programme" funded by the European Union.

\section{REFERENCES}

[1] Z.P. Bažant and A. Steffens, "Mathematical model for kinetics of alkali-silica reaction in concrete", Cement and Concrete Research 30, 419-428 (2000).

[2] A. Winnicki and S. Pietruszczak, "On mechanical degradation of reinforced concrete affected by alkali-silica reaction", J. Engineering Mechanics 134 (8), 611-626 (2008).

[3] F.J. Ulm, O. Coussy, L. Kefei, and C. Larive, "Thermo-chemomechanics of ASR expansion in concrete structures", J. Engineering Mechanics 126, 233-242 (2000).

[4] C. Comi, R. Fedele, and U. Perego, "A chemo-thermo-damage model for the analysis of concrete dams affected by alkali-silica reaction", Mechanics of Materials 41 (3), 210-230 (2009).

[5] B.A. Schrefler, G. Khoury, D. Gawin, and C.E. Majorana, "Thermo-hydro-mechanical modelling of high performance concrete at high temperatures", Eng. Computations 19 (7), 787-819 (2002).

[6] D. Gawin, F. Pesavento, and B.A. Schrefler, "Modelling of hygro-thermal behaviour of concrete at high temperature with thermo-chemical and mechanical material degradation", Computer Methods in Applied Mechanics and Eng. 192, 1731-1771 (2003).

[7] D. Gawin, F. Pesavento, and B.A. Schrefler, "Hygro-thermochemo-mechanical modelling of concrete at early ages and beyond. Part II: Shrinkage and creep of concrete", Int. J. Numerical Methods in Eng. 67, 332-363 (2006).

[8] F. Pesavento, D. Gawin, and B.A. Schrefler, "Modeling cementitious materials as multiphase porous media: theoretical framework and applications", Acta Mechanica 201, 313-339 (2008).

[9] D. Gawin, F. Pesavento, and B.A. Schrefler, "Modeling of cementitious materials exposed to isothermal calcium leaching, with considering process kinetics and advective water flow. Part 1: Theoretical model", Int. J. Solids and Structures 45 (25-26), 6221-6240 (2008).

[10] D. Gawin, F. Pesavento, and B.A. Schrefler, "Modeling of cementitious materials exposed to isothermal calcium leaching, with considering process kinetics and advective water flow. Part 2: Numerical solution", Int. J. Solids and Structures 45 (25-26), 6241-6268 (2008).

[11] M. Koniorczyk and D. Gawin, "Heat and moisture transport in porous building materials containing salt", J. Building Physics 31 (4), 279-300 (2008).

[12] D. Gawin, F. Pesavento, and B.A. Schrefler, "Modeling deterioration of cementitious materials exposed to calcium leaching in non-isothermal conditions", Computer Methods in Applied Mechanics and Eng. 198 (37-40), 3051-3083 (2009).

[13] M. Koniorczyk, "Modelling the phase change of salt dissolved in pore water - equilibrium and non-equilibrium approach", Construction and Building Materials 24, 1119-1128 (2010).

[14] M. Koniorczyk and D. Gawin, "Numerical modeling of salt transport and precipitation in non-isothermal partially saturat- 
ed porous media considering kinetics of salt phase changes", Transport in Porous Media 87 (1), 57-76 (2011).

[15] M. Koniorczyk, "Salt transport and crystallization in nonisothermal, partially saturated porous materials considering ions interaction model", Int. J. Heat and Mass Transfer 55, 665-679 (2012).

[16] D. Gawin, M. Lefik, and B.A. Schrefler, "ANN approach to sorption hysteresis within a coupled hygro-thermo-mechanical FE analysis", Int. J. Numerical Methods in Eng. 50, 299-323 (2001).

[17] J. Bear and Y. Bachmat, Introduction to Modeling of Transport Phenomena in Porous Media, Kluwer Academic Publishers, Amsterdam, 1991.

[18] R.W. Lewis and B.A. Schrefler, The Finite Element Method in the Static and Dynamic Deformation and Consolidation of Porous Media, 2nd ed., John Wiley \& Sons, Chichester, 1998.

[19] O. Coussy, Poromechanics, John Wiley \& Sons, Chichester, 2004.

[20] S.M. Hassanizadeh and W.G. Gray, "General conservation equations for multi-phase systems: 1. averaging procedure", Advances in Water Resources 2, 131-144 (1979).

[21] S.M. Hassanizadeh and W.G. Gray, "General conservation equations for multi-phase systems: 2. mass, momenta, energy and entropy equations", Advances in Water Resources 2, 191-203 (1979).

[22] S.M. Hassanizadeh and W.G. Gray, "General conservation equations for multi-phase systems: 3 . constitutive theory for porous media flow", Advances in Water Resources 3, 25-40 (1980).

[23] M. Wyrzykowski, P. Lura, F. Pesavento, and D. Gawin, "Modeling of internal curing in maturing mortar", Cement and Concrete Research 41 (12), 1349-1356 (2011).

[24] D. Gawin, Modelling of Coupled Hygro-Thermal Phenomena in Building Materials and Building Components. Scientific Bulletin 853, Publishing House of Łódź Technical University, Łódź, 2000, (in Polish).

[25] R.M. Espinosa, L. Franke, and G. Deckelmann, "Phase changes of salts in porous materials: crystallization, hydration and del- iquescence", Construction and Building Materials 22, 1758 1773 (2008).

[26] M. Koniorczyk and P. Konca, "Salt and moisture transport in non-isothermal condition considering the kinetics of salt phase change", Proc. 12th Int. Conf. on Durability of Building Materials and Components 1, 439-446 (2011).

[27] M. Steiger, "Crystal growth in porous materials - I. The crystallization pressure of large crystals", J. Crystal Growth 282, 455-469 (2005).

[28] K.S. Pitzer, "Ion interaction approach: theory and data correlation", in Activity Coefficients in Electrolyte Solutions, 2nd ed., ed. K.S. Pitzer, pp.75-153, CRC Press, New York, 1991.

[29] W.G. Gray, B.A. Schrefler, and F. Pesavento, "The solid phase stress tensor in porous media mechanics and the Hill-Mandel condition", J. Mech Phys Solids 57, 539-554 (2009).

[30] D. Gawin, F. Pesavento, and B.A. Schrefler, "Modelling creep and shrinkage of concrete by means of effective stresses", $M a$ terials and Structures 40 (6), 579-591 (2007).

[31] G.W. Scherer, "Crystallization in pores", Cement and Concrete Research 22, 1347-1358 (1999).

[32] O.C. Zienkiewicz and R.L. Taylor, The Finite Element Method. The Basis, vol. 1, Butterworth-Heinemann, Oxford, 2000.

[33] M. Koniorczyk and D. Gawin, "Modelling of salt crystallization in building materials with microstructure - poromechanical approach", Construction and Building Materials 36, 860873 (2012).

[34] P. Rucker, M. Krus, and A. Holm, "Einsatz einer Kombinierten Messtechnikmethode zur Untersuchung von Salztransportvergangen", Bauphysik 25, 296-302 (2003), (in German).

[35] R. Cerny, Z. Pavlik, and P. Rovnanikova, "Experimental analysis of coupled water and chloride transport in cement mortar", Cement and Concrete Composites 26, 705-715 (2004).

[36] A.M. Neville, Properties of Concrete, 4th edition, Longman House, Harlow, 1995.

[37] V. Baroghel-Bouny, M. Mainguy, T. Lassabatere, and O. Coussy, "Characterization and identification of equilibrium and transfer moisture properties for ordinary and high-performance cementitious materials", Cement and Concrete Research 29, 1225-1238 (1999). 